and sex of participants $(p=0.591)$ were found not to be significantly related to CMV IgM sero-positivity.

Conclusion Cytomegalovirus is hyperendemic among HIV seropositive and sero-negative individuals in Ilorin. This suggests primarily a horizontal, non sexual route of transmission. There is need for multi-centre studies using more sensitive tests such as CMV DNA PCR in order to establish the prevalence of CMV infection among HIV/AIDS patients.

\section{P3.189 HIV/STI BIO-BEHAVIOURAL CHARACTERISTICS OF KEY POPULATIONS AT HIGHER RISK}

doi:10.1136/sextrans-2013-051184.0646

S Grigoryan, A Papoyan, A Hakobyan, T Grigoryan, R Hovhannisyan, T Balayan. National AIDS Center, Yerevan, Armenia

Background From 2002 bio-behavioural surveillance has regularly conducted in Armenia to monitor HIV prevalence and behavioural characteristics among the key populations at higher risk.

In 2010 and 2012 integrated HIV/STI/Hepatitis biological and behavioural surveillance was conducted among persons who inject drugs (PWID), sex workers (SWs) and men who have sex with men (MSM) in three major cities of Armenia.

Methods Respondent driven sampling was used to conduct the surveillance in 2010 and 2012. 300 representatives of each population group participated in the surveillance surveys conducted in 2012 in Yerevan, the capital. STI component aimed to reveal risk behaviours recently exhibited by the respondents.

Results The biological and behavioural surveillance showed that HIV prevalence among PWID in Yerevan was 6.3\%, syphilis prevalence was $0.8 \%$, and hepatitis C prevalence $-52.6 \%$. $55 \%$ of PWID did not use condoms at last sex with casual partners, $29.1 \%$ of those surveyed shared injecting equipment in the last 1 month.

HIV prevalence among SWs was $1.3 \%$, syphilis prevalence $4.3 \%$, trichomoniasis prevalence - $22.5 \%$, gonorrhoea prevalence $6.6 \% .10 \%$ of SWs did not use condoms at last sex with clients and only $40 \%$ used condoms at last sex with non-commercial partners. HIV prevalence among MSM was $2.6 \%$, syphilis prevalence $-1.9 \%$, and hepatitis B prevalence $-0.6 \%$. $27 \%$ of MSM did not use condoms at last sex with casual partners.

Conclusion The surveillance results revealed that representatives of the key populations at higher risk continue to exhibit risk behaviours, which is proved by low condom use. Also, presence of sexually transmitted infections demonstrates their risk behaviours. Bio-behavioural surveys with the same methodology would be continued for making the trend analysis. The survey results should be considered while designing prevention activities. The study outcomes should be disseminated among stakeholders for better planning and among communities for advocacy.

\section{P3.190 EPIDEMIOLOGY OF HIV INFECTION BEFORE AND AFTER RISING THE IRON CURTAIN: THE HUNGARIAN EXPERIENCE}

doi:10.1136/sextrans-2013-051184.0647

${ }^{1}$ K Nagy, 'S Kanizsai, ${ }^{2}$ V Varkonyi, ${ }^{3} \mathrm{~J}$ Aradi. ${ }^{1} / n s t$. Medical Microbiology, Semme/weis University, Budapest, Hungary; ${ }^{2}$ Korut privat Praxis, Budapest, Hungary; ${ }^{3}$ Dept. Biochemistry, Univ.Debrecen, Debrecen, Hungary

Background In the beginning of 21st Century Hungary has a good epidemiological situation concerning HIV infections and AIDS which was the result of the strict but consistent introduction of nationwide HIV screening in 1985, and the good cooperation of clinicians, researchers and government officials.

Methods HIV subtypes and genetic resistance (allele frequencies of CCR5- $\Delta 32$, CCR2-64I and SDF1-3'A) were determined by molecular virological methods, antiretroviral drug resistance in primary HIV infection and transmission of HIV CRFs by illegal migrants were analysed by genotyping (Truegene HIV-1 Genotyping System/Siemens/). New approach in inhibiting HIV infection on cellular level by modifying SH- groups of CD4 and HIV gp120 env by novel thyolated nucleosides were developed using viral pseudotypes.

Results In the last 28 years MSM is still the highest risk group, while HIV infection is low in i.v. drug users. In the early years HIV $\mathrm{B}$ subtypes were determined as major circulating variants. CCR5$\Delta 32$ was found in $12 \%$ of general population and $15 \%$ in ethnic gypsy minority. Genotyping revealed, that approx. $15 \%$ of primary HIV infections are transmitted by drug resistant mutants mainly in MSMs. After 2004, when Hungary joined to the European Union illegal migration increased, besides the prostitutes mainly from SE Asia and Africa, resulting in the appearance of new HIV subtypes and African CRFs, such as CRF02_AG (28.5\%), CRF06_cpx (17.8\%) and CRF11_cpx(3.6\%). Novel entry inhibitor UD was effective in vitro in $5 \mathrm{uM}$

Discussion Transmission of drug-resistant HIV during primary infection, penetration of African CRFs raise serious clinical and public health consequences. To maintain the recent favourable epidemiological situation screening programmes should be continued, HIV genotyping at the time of diagnosis should be the standard of care, and introduction of novel compounds, such as entry inhibitors in the treatment are necessary.

Supported by OTKA 81367 Grant.

\section{P3.191 DO GAINS IN THE PREVENTION OF HIV LEAD TO LOSSES IN THE PREVENTION OF OTHER SEXUALLY TRANSMITTED INFECTIONS?}

doi:10.1136/sextrans-2013-051184.0648

${ }^{1,2}$ C Rietmeijer, ' $\mathrm{C}$ Mettenbrink, ${ }^{1} \mathrm{~A}$ Al-Tayyib, 'M Thrun. 'Denver Public Health Department, Denver, CO, United States; ${ }^{2}$ Colorado School of Public Health, Aurora, CO, United States

Background Aggressive efforts to reduce the viral load among persons with HIV infection, including early diagnosis, linkage to care, and early initiation of antiretroviral treatment may significantly reduce HIV transmission at the community level, and thus becoming a major focus of HIV prevention policy. However, it is not known how this approach will affect HIV risk behaviours and the epidemiology of non-HIV sexually transmitted infections (STI).

Objective To study trends in HIV, early syphilis, and high-risk behaviours among men who have sex with men (MSM) in Denver. Methods We analysed and triangulated data from 3 sources: the Denver Metro Health (STI) Clinic; Denver STI surveillance; Thee Denver MSM waves of the National HIV Behavioral Surveillance system (NHBS).

Results Among MSM visiting DMHC, the rate of new HIV diagnoses decreased from $3.3 \%$ in 2007 to $2.5 \%$ in 2012 (-24\%) while the rate of early syphilis increased from $1.3 \%$ to $2.5 \%$ (+92\%) during the same time period. Similarly, in Denver (all populations), rates of newly reported HIV infections decreased from 32.4/100,000 in 2007 to $25.3 / 100,000$ in 2011 ( $-22 \%$ ), but newly reported cases of primary and secondary syphilis increased from 5.3/100,000 to $11.8 / 100,000(+123 \%)$ during the same time period. Finally, Denver NHBS data showed the proportion of MSM reporting unprotected anal sex stable at 36\%-37\% between 2005, 2009 and 2011 data waves.

Conclusion Despite continued high levels of risk behaviours among Denver MSM, the rate of new HIV infections is decreasing, providing evidence for the effectiveness of efforts to reduce the community HIV viral load. However, at the same time, rates of early syphilis continue to rise. Without simultaneous efforts to reduce risk behaviours, a biomedical approach to reduce the community HIV viral load may result in gains for HIV prevention but losses in the prevention of syphilis and other STI. 